\title{
Novel risk calculator performance in athletes with arrhythmogenic right ventricular cardiomyopathy $e$
}

\author{
Alessio Gasperetti, MD, ${ }^{* \dagger 1}$ Antonio Dello Russo, MD, PhD, ${ }^{\ddagger 1}$ Mattia Busana, MD, ${ }^{\circ}$ \\ Mariantonietta Dessanai, MD, ${ }^{*}$ Francesca Pizzamiglio, MD, * \\ Ardan Muammer Saguner, MD, ${ }^{\dagger}$ Anneline S.J.M. te Riele, MD, PhD, \\ Elena Sommariva, PhD, ${ }^{*}$ Giulia Vettor, MD, PhD, ${ }^{*}$ Laurens Bosman, MD, \\ Firat Duru, MD, ${ }^{\dagger}$ Paolo Zeppilli, MD, ${ }^{\| * *}$ Luigi Di Biase, MD, PhD, ${ }^{\dagger \dagger}$ Andrea Natale, MD, ${ }^{\ddagger \ddagger}$ \\ Claudio Tondo, MD, PhD, ${ }^{\star} \S \S$ Michela Casella, MD, PhD* \\ From the *Heart Rhythm Center, Centro Cardiologico Monzino, IRCCS, Milan, Italy, ${ }^{\dagger}$ Department of \\ Cardiology, University Hospital of Zurich, Zurich, Switzerland, ${ }^{\ddagger}$ Cardiology and Arrhythmology Clinic, \\ Marche Polytechnic University, University Hospital "Ospedali Riuniti, "Ancona, Italy, ${ }^{\S}$ Department of \\ Anesthesiology, Emergency and Intensive Care Medicine, University of Göttingen, Göttingen, \\ Germany, "Department of Heart and Lungs, Division of Cardiology, University Medical Center \\ Utrecht, Utrecht, The Netherlands, "Istituto di Cardiologia, Università Cattolica del Sacro Cuore \\ Rome, Rome, Italy, **Istituto di Medicina Dello Sport, Università Cattolica del Sacro Cuore, \\ Fondazione Policlinico Universitario Agostino Gemelli IRCCS, Rome, Italy, ${ }^{\dagger \dagger}$ Montefiore-Einstein \\ Center for Heart and Vascular Care, Montefiore Medical Center, Albert Einstein College of Medicine, \\ Bronx, New York, ${ }^{\ddagger}$ Texas Cardiac Arrhythmia Institute at St. David's Medical Center, Austin, Texas, \\ and ${ }^{\S \S}$ Department of Clinical Science and Community Health, University of Milan, Milan, Italy.
}

BACKGROUND Disease progression and ventricular arrhythmias (VAs) in arrhythmogenic right ventricular cardiomyopathy (ARVC) are correlated with physical exercise, and clinical detraining and avoidance of competitive sport practice are suggested for ARVC patients. An algorithm assessing primary arrhythmic risk in ARVC patients was recently developed by Cadrin-Tourigny et al. Data regarding its transferability to athletes are lacking.

OBJECTIVE The purpose of this study was to assess the reliability of the Cadrin-Tourigny risk prediction algorithm in a cohort of athletes with ARVC and to describe the impact of clinical detraining on disease progression.

METHODS All athletes undergoing clinical detraining after ARVC diagnosis at our institution were enrolled. Baseline and follow-up clinical characteristics and data on VA events occurring during follow-up were collected. The Cadrin-Tourigny algorithm was used to calculate the a priori predicted VA risk, which was compared with the observed outcomes.

RESULTS Twenty-five athletes (age $36.1 \pm 14.0$ years; $80 \%$ male) with definite ARVC who were undergoing clinical detraining were enrolled. Over median (interquartile range) follow-up of 5.3 (3.2-6.6) years, a reduction in premature ventricular complex (PVC) burden $(P=.001)$ was assessed, and 10 VA events $(40 \%)$ were recorded. The a priori algorithm-predicted risk seemed to fit with the observed cohort arrhythmic risk [mean observedpredicted risk difference over 5 years $-0.85 \%$ (interquartile range $-4.8 \%$ to $+3.1 \%) ; P=.85$ ]. At 1 -year follow-up, 11 patients (44\%) had an improved stress ECG response, and no significant changes in right ventricular ejection fraction were observed.

CONCLUSION Clinical detraining is associated with PVC burden reduction in athletes with ARVC. The novel risk prediction algorithm does not seem to require any correction for its application to ARVC athletes.

KEYWORDS Arrhythmogenic right ventricular cardiomyopathy; Athletes; Clinical detraining; Physical exercise; Risk calculator; Ventricular arrhythmia

(Heart Rhythm 2020; $\mathbf{\square : 1 - 9 ) ~ ( c ) ~} 2020$ Heart Rhythm Society. All rights reserved.

Dr Tondo received modest honoraria from St. Jude Medical and Abbott; and serves as member of the advisory board for Medtronic Inc. and Boston Scientific Corp. Dr Di Biase is a consultant for Biosense Webster, Boston Scientific, and St. Jude Medical; and has received modest honoraria from Medtronic, AtriCure, and EPiEP. Dr Natale is a consultant for Boston Scientific, Biosense Webster, St. Jude Medical, Biotronik, and Medtronic. All other authors have reported that they have no conflicts relevant to the contents of this paper to disclose. ${ }^{1} \mathrm{Dr}$ Alessio Gasperetti and Dr Antonio Dello Russo contributed equally to this work. Address reprint requests and correspondence: Dr Alessio Gasperetti, Heart Rhythm Center, Centro Cardiologico Monzino, IRCCS, Via Carlo Parea 4, 20100 Milan, Italy. E-mail address: alessio.gasperetti93@gmail.com. 


\section{Background}

Arrhythmogenic right ventricular cardiomyopathy (ARVC) is an inherited cardiomyopathy characterized by predominant but not exclusive right ventricular (RV) myocardial fibrofatty replacement. ${ }^{1-3}$ It usually is characterized by electrical and morphologic RV alterations that are well known to be associated with ARVC and an important increase in sudden cardiac death (SCD) risk due to malignant ventricular arrhythmias (VAs). ${ }^{1,3}$ Implantable cardioverterdefibrillators (ICDs) are a viable option for dealing with SCD risk in this patient population, ${ }^{4}$ but until recently, appropriate tools for adequate individual primary arrhythmic risk stratification were scarce. ${ }^{5-8}$ Recently, a novel algorithm for primary prevention ICD placement was developed based on a large sample size international registry. ${ }^{9}$ Its performance seems to be superior to that of the 2015 International Task Force (ITF) consensus statement criteria, possibly leading to better patient-tailored therapy and increased net clinical benefit.

Several studies have assessed the correlation between ARVC disease progression and malignant VA genesis with engagement in sports. ${ }^{10-14}$ Physical exercise greatly increases arrhythmic risk in such patients, and appropriate detraining and sport practice reduction after disease diagnosis reduce the long-term risk of VAs. ${ }^{15}$ No specific adaptation of the current Cadrin-Tourigny et al algorithm (https://arvcrisk.com) for individual levels of physical exercise has been provided. Data for validation of this algorithm in athletes are lacking, and transferability of the algorithm to an external high-intensity athlete cohort with ARVC has yet to be tested.

The aims of this study were to validate the CadrinTourigny et al algorithm and to report on disease progression in a well-characterized, high-intensity athlete cohort of patients with ARVC.

\section{Methods}

\section{Patient population}

All consecutive athletes with a definitive ARVC diagnosis evaluated at the Arrhythmology and Sport Medicine Unit of IRCCS Centro Cardiologico Monzino were enrolled in the study. The analysis was approved by the local ethic review board according to center's legislation and complied with the Declaration of Helsinki.

\section{Cohort definition}

ARVC diagnoses were made by a dedicated heart team composed of cardiac radiologists, electrophysiologists, and cardiac pathologists, in accordance with the 2010 Revised Task Force Criteria. ${ }^{16}$

A patient was classified as an athlete after a complete sport cardiology evaluation according to the patient's training regimen: $>6$ hours per week of sports activity with moderate-to-intense dynamic components (at least 6 METs); affiliation with an Italian or internationally recognized sport federation; regular participation in sport competitions over the past year; or history of sport activity without significant breaks/changes in training pattern/load over the past 3 years. ${ }^{15,17}$ Endurance sports were defined as those requiring sustained effort at $>70 \% \mathrm{VO}_{2} \mathrm{max}$ (eg, cycling, swimming, rowing). Mixed sports were defined as those requiring a mix of skill-based and aerobic/anaerobic exercise (eg, football, basketball, volleyball). ${ }^{18}$ All patients regularly practiced their sport up until the time of disease diagnosis or until their eligibility for participation in the sport was suspended immediately before referral to our center.

\section{Patient in-hospital evaluation}

After referral from the outpatient clinic to our centers or during hospitalization after direct admission, all enrolled athletes routinely underwent 12-lead baseline electrocardiography (ECG), 24-hour Holter ECG monitoring, complete blood panel, stress ECG test, ARVC dedicated cardiac ultrasound, and cardiac magnetic resonance (CMR) analysis.

Baseline programmed electrical stimulation was performed routinely as part of the arrhythmic risk assessment. Three-dimensional endocavitary electroanatomic mapping (EAM), percutaneous EAM-guided endomyocardial biopsy, and genetic testing for diagnostic purposes were performed based on indication and physician expertise. SCD risk stratification analyses were performed in all patients per the ITF consensus statement for ARVC treatment, ${ }^{4}$ and ICD placement was performed accordingly.

\section{Evaluation of arrhythmic events and patient follow-up}

Upon disease diagnosis, 24-hour premature ventricular complex (PVC) burden and all complex arrhythmic events (PVCs, sustained ventricular tachycardia [SVT], ventricular fibrillation/flutter $[\mathrm{VF}]$ ) at baseline and noted in the patient history were recorded, as was the reason for referral to our center. At disease diagnosis, the patient's sport eligibility status was immediately suspended. Patients were instructed to start a detraining period that highly reducing their training regimen and to abstain from competitive sport practice per international sport medicine guidelines. ${ }^{19,20}$

Patients underwent follow-up for disease progression 6 months after hospital discharge and then every 12 months thereafter, or immediately after the occurrence of an arrhythmic event and/or an emergency room visit. Holter ECG was required at 6 months, with a surveillance protocol that required a minimum of one 24-hour Holter recording every 24 months; however, monitoring protocols were individualized based on physician expertise and patient availability. For patients with an ICD, an ICD interrogation was performed by dedicated personnel every 6-8 months, and a summary check was performed contextually at every follow-up visit. ECG stress test, cardiac ultrasound, and CMR follow-up surveillance were not mandated per protocol. 


\section{Study outcomes}

The primary outcome of the study was the first sustained VA event after disease diagnosis. Sustained VAs were defined as a composite of SCD, SVT, VF, or appropriate ICD intervention for any of the previous arrhythmias.

For all patients who complied with the model requirements (as reported in the referred study and on the https:// arvcrisk.com Web site), ${ }^{9}$ the expected VA rate was calculated using the Cadrin-Tourigny et al predictive model. Yearly risk was calculated at the time of disease diagnosis using the values of the indicated variables as measured at disease diagnosis. Observed sustained VA rate at long-term follow-up was calculated and compared to the algorithm-predicted rate. Data on changes in 24-hour Holter ECG PVC number, stress ECG result, and right ventricular ejection fraction (RVEF) modification before and after the detraining protocol also were collected and analyzed as secondary outcomes.

\section{Statistical analysis}

All statistical analyses were performed using R Project for statistical computing Version 3.5. Continuous variables are expressed as mean $\pm \mathrm{SD}$ or median (interquartile range $[\mathrm{IQR}])$. Categorical variables are expressed as count (\%). Comparisons were performed using the independent sample Student $t$ test or the Mann-Whitney U test, as appropriate. Kaplan-Meier analysis with log-rank test was used for statistical inference of long-term outcome data. Predicted and observed frequencies of sustained VAs were evaluated and compared using a multivariate linear regression model. To evaluate the effect of time over the total number of PVCs and on RVEF, we fit a mixed model to the data in which time was considered the fixed effect and the subject was the random effect. The model ignores the missing data but generates an output with the same observation number as in the original dataset. The findings were considered statistically significant with a 2 -tailed $P<.05$.

\section{Results}

\section{Cohort overview}

A total of 25 athletes with a definitive ARVC diagnosis were enrolled in the study, with men representing the majority $(80 \%)$. Mean age at disease diagnosis was $36.1 \pm 14.0$ years. All patients were probands; 6 (24\%) had a history of SCD in their family at in-depth evaluation. Fourteen patients (56\%) practiced an aerobic/anaerobic mixed sport, and the remaining 11 patients $(44 \%)$ were endurance athletes.

The most common reason for referral was the presence of abnormalities at a sport eligibility assessment $(\mathrm{n}=18$ [72\%]), followed by a symptomatic VA index event $(\mathrm{n}=5$ [20\%]), 2 of which required emergency resuscitation maneuvers on the field due to sudden cardiac arrest. At first evaluation, the median number of PVCs on 24-hour Holter ECG was 1000 (300-3500). Seven patients (28\%) had a history of documented nonsustained ventricular tachycardia, and 5 (20\%) had documented sustained VAs. At CMR, mean RVEF was $47.5 \% \pm 8.1 \%$, and mean left ventricular EF
Table 1 Patient cohort baseline characteristics $(\mathrm{N}=25)$

\begin{tabular}{|c|c|}
\hline \multicolumn{2}{|l|}{ Demographics } \\
\hline Age at diagnosis $(\mathrm{y})$ & $36.1 \pm 14.0$ \\
\hline Male & $20(80.0)$ \\
\hline Training load (h) & $7.4 \pm 1.3$ \\
\hline \multicolumn{2}{|l|}{ Sport practice } \\
\hline Mixed sports & $14(56.0)$ \\
\hline Volley & $3(12.0)$ \\
\hline Football & $7(28.0)$ \\
\hline Basket & $4(16.0)$ \\
\hline Endurance sports & $11(44.0)$ \\
\hline Cycling & $5(20.0)$ \\
\hline Endurance running & $4(16.0)$ \\
\hline Triathlon & $1(4.0)$ \\
\hline Rowing & $1(4.0)$ \\
\hline \multicolumn{2}{|l|}{ Reason for referral } \\
\hline Abnormalities at sport eligibility visit & $18(72)$ \\
\hline ECG static abnormalities & $5(20.0)$ \\
\hline PVCs & $8(32.0)$ \\
\hline NSVT & $5(20.0)$ \\
\hline Complex VAs during PA & $5(20)$ \\
\hline SVT & $3(12.0)$ \\
\hline VF with SCD & $2(8.0)$ \\
\hline Unexplained loss of consciousness & $2(8)$ \\
\hline \multicolumn{2}{|l|}{ Stress test ECG } \\
\hline Negative & $6(24.0)$ \\
\hline PVCs suppressed by PA & $10(40.0)$ \\
\hline PVCs unsuppressed by PA & $5(20.0)$ \\
\hline NSVT & $3(12.0)$ \\
\hline SVT & $1(4.0)$ \\
\hline \multicolumn{2}{|l|}{ CMR imaging } \\
\hline $\operatorname{RVEF}(\%)$ & $47.5 \pm 8.1$ \\
\hline $\operatorname{LVEF}(\%)$ & $56.3 \pm 5.2$ \\
\hline \multicolumn{2}{|l|}{ Interventions } \\
\hline PES inducibility & $9(36.0)$ \\
\hline SVT & $7(28.0)$ \\
\hline VF & $2(8.0)$ \\
\hline ICD placement & $10(40.0)$ \\
\hline Subcutaneous ICD & $4(16.0)$ \\
\hline Transvenous dual-chamber ICD & $5(20.0)$ \\
\hline Transvenous single-chamber ICD & $1(4.0)$ \\
\hline
\end{tabular}

Values are given as mean \pm SD or $n(\%)$.

CMR = cardiac magnetic resonance; ECG = electrocardiography; ICD $=$ implantable cardioverter-defibrillator; $\mathrm{LVEF}=$ left ventricular ejection fraction; NSVT = nonsustained ventricular tachycardia; PA = physical activity; $\mathrm{PES}=$ programmed electrical stimulation; $\mathrm{PVC}=$ premature ventricular complex; RVEF = right ventricular ejection fraction; $S C D=$ sudden cardiac death; SVT = sustained ventricular tachycardia; VA = ventricular arrhythmia; $\mathrm{VF}=$ ventricular fibrillation.

was $56.3 \% \pm 5.2 \%$. Genetic testing analysis in 15 athletes $(60 \%)$ revealed 9 were positive for a pathogenic or likely pathogenic mutation.

All patients underwent programmed electrical stimulation. Nine patients $(26 \%)$ had VAs induced in the electrophysiology laboratory. EAM and EAM-guided endomyocardial biopsy were performed in 10 patients $(40 \%)$. After disease diagnosis and appropriate arrhythmic risk stratification, 10 ICD implantations (4 subcutaneous, 5 transvenous dual-chamber, 1 transvenous single-chamber) (40.0\%) were performed; an additional S-ICD placement was deemed necessary but was refused by the patient. The characteristics of the study population are given in Table 1 . 


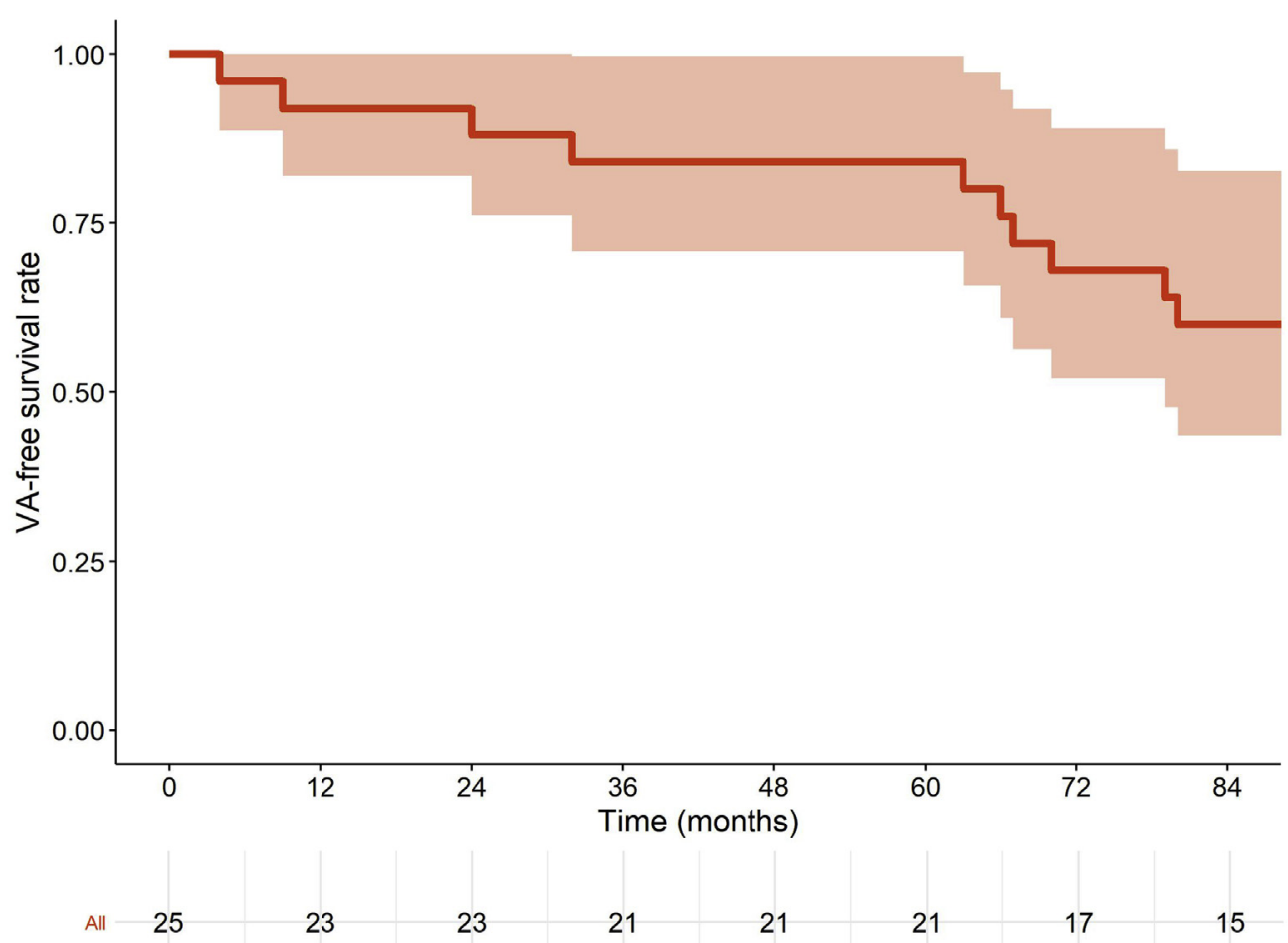

Figure 1 Overall freedom from ventricular arrhythmias (VAs) in the overall cohort.

\section{Follow-up arrhythmic analysis}

Over median follow-up of 5.3 (3.2-6.6) years, a sustained VA event was documented in 10 athletes (40.0\%). Of these episodes, 6 were recorded in patients with a previously implanted ICD. The cumulative freedom from VA events of the cohort is shown in Figure 1 [overall 5-year freedom from VA rate $0.84(0.71-0.92)]$.

Within the cohort, 20 patients $(80 \%)$ complied with the Cadrin-Tourigny et al algorithm requirements, and their predicted risk was calculated. The clinical characteristics of this subpopulation are given in Supplemental Table 1. Comparison between predicted and observed VA rates over time are shown in Figure 2. Events at follow-up were well within the 95\% confidence interval of predicted rates, with a nonsignificant difference from the algorithm-predicted rate at any time during follow-up [mean difference observed-predicted rate over 5 years $-0.85 \%$ ( $-4.80 \%$ to $+3.10 \%) ; P=.85]$. The entire risk data analysis is given in Supplemental Table 2.

\section{Disease progression during follow-up}

After disease diagnosis and the beginning of the detraining protocol, the characteristics of the athletes were collected over time to monitor disease evolution. Over time, a statistically significant 24 hour/PVC count reduction was observed $(P=.001)$ (mean reduction over first 18 months $-1682 \pm 573 ; P=.048$; mean reduction from 18 months to end of follow-up $160 \pm 680 ; P=.99$ ) (Figure 3). A subanalysis revealed that the presence of beta-blocker did not significantly influence the effect of clinical detraining on the 24 hour/PVC count $(P=.33)$ (Figure 4).
Over a median of 8 (4-11) months, 21 patients (84\%) underwent a repeat stress ECG. A per patient qualitative improvement in arrhythmic response at stress testing was observed in 11 patients (52\%), the same arrhythmic response in $9(43 \%)$, and a worsened arrhythmic stress ECG response in only $1(5 \%)$ (patient did not follow the detraining protocol). Graphic representation of stress ECG data over time is shown in Figure 5. A total of 18 patients $(72 \%)$ repeated CMR examination after a median of 12 (9-13) months, and RVEF had remained stable [CMR RVEF mean change $+0.11 \%(-2.31 \%$ to $+2.54 \%) ; P=.92$; mean change excluding 2 patients who did not follow the detraining proto$\mathrm{col}+1.43 \%(-0.23 \%$ to $+3.11 \%) ; P=.09$ ] (Figure 6 ).

\section{Discussion}

ARVC is the first cause of sudden death among young individuals, and the risk has been reported as increased 5-fold in active athletes, ${ }^{10,21}$ specifically in those training with a high-intensity regimen and participating in endurance sport competitions. ${ }^{3,10}$ Physical activity has been demonstrated to increase disease progression and overall arrhythmic risk in this patient population, ${ }^{11-14,22,23}$ with high-end strenuous exercise and endurance training associated with the worst outcomes. ${ }^{24}$ Given these findings, the current sport medicine guidelines suggest complete abstention from competitive sport training and a significant reduction even in leisure sport activities by ARVC patients. ${ }^{19,20}$

Current scientific consensus indicates ICD placement in high arrhythmic patients after arrhythmic risk assessment to be the most effective treatment for ARVC patients. ${ }^{4}$ 


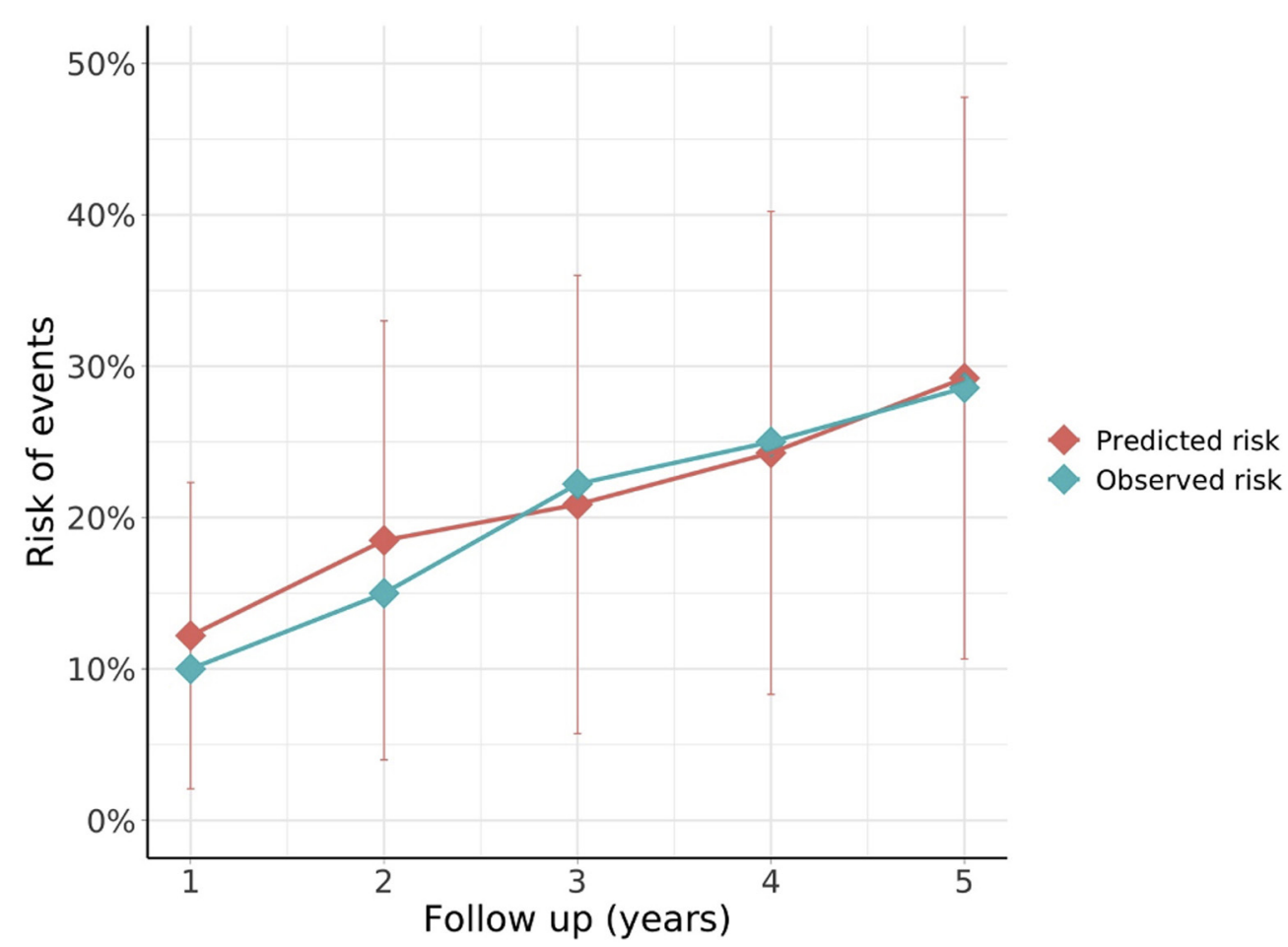

Figure 2 Comparison between observed and algorithm-predicted event rates. The algorithm-predicted risk showed almost perfect adherence to observed event rates over follow-up. Time is considered from disease diagnosis.

However, a high rate of ICDs implanted per VAs treated has been reported in the ARVC literature, and due to devicerelated economic burden and risks, a refinement of arrhythmic stratification strategies was needed. ${ }^{9}$ In a recent study, Cadrin-Tourigny et $\mathrm{al}^{9}$ presented a novel arrhythmic risk prediction model (https://arvcrisk.com) that proved effective in an external experience. ${ }^{25}$ To date, no specific correction for physical activity has been proposed.

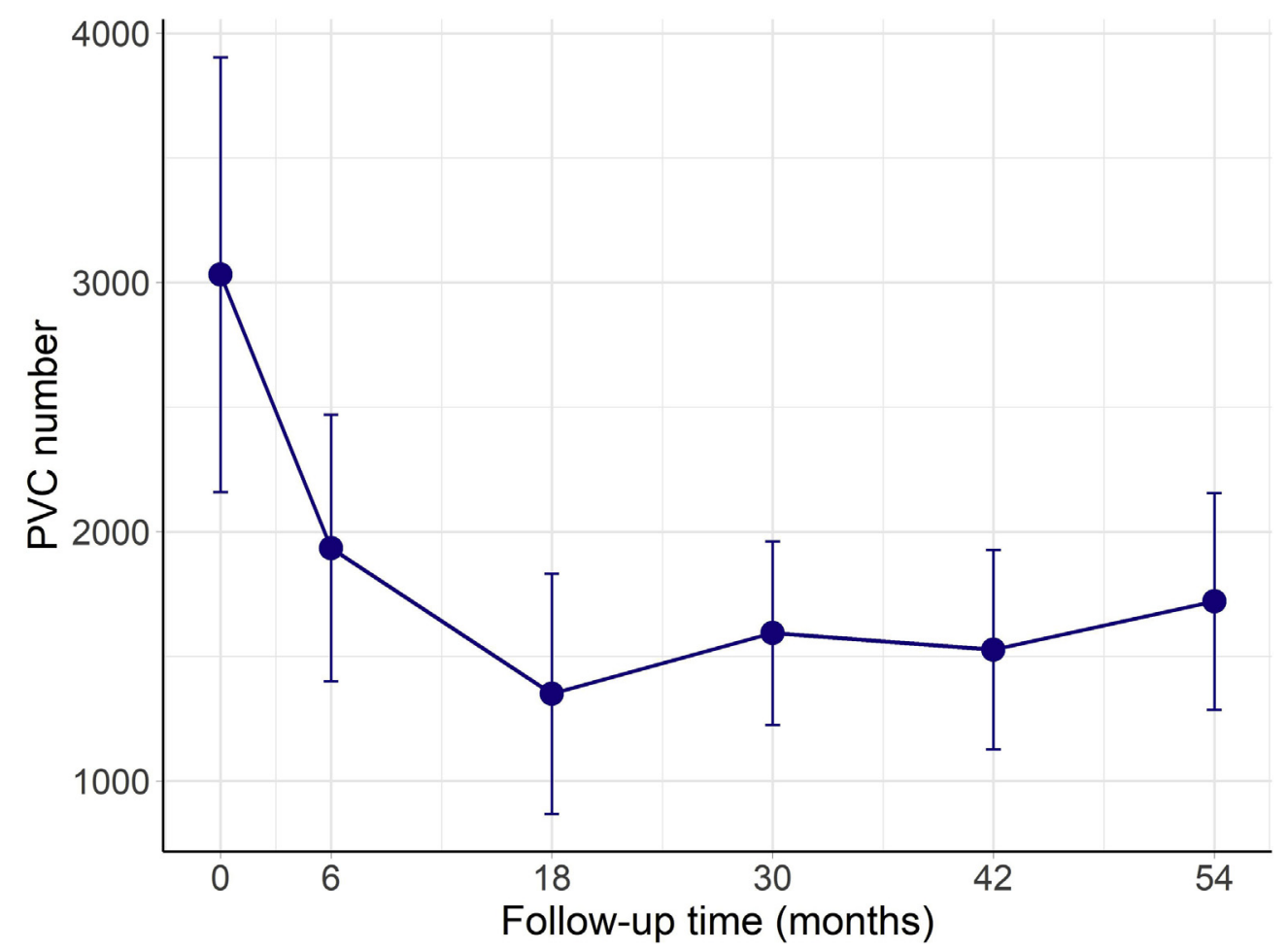

Figure 3 Mean premature ventricular contraction (PVC)/24 hours over follow-up for the entire cohort. A clear reduction in PVC number can already be observed at 6 months from detraining. Time is considered from disease diagnosis. 


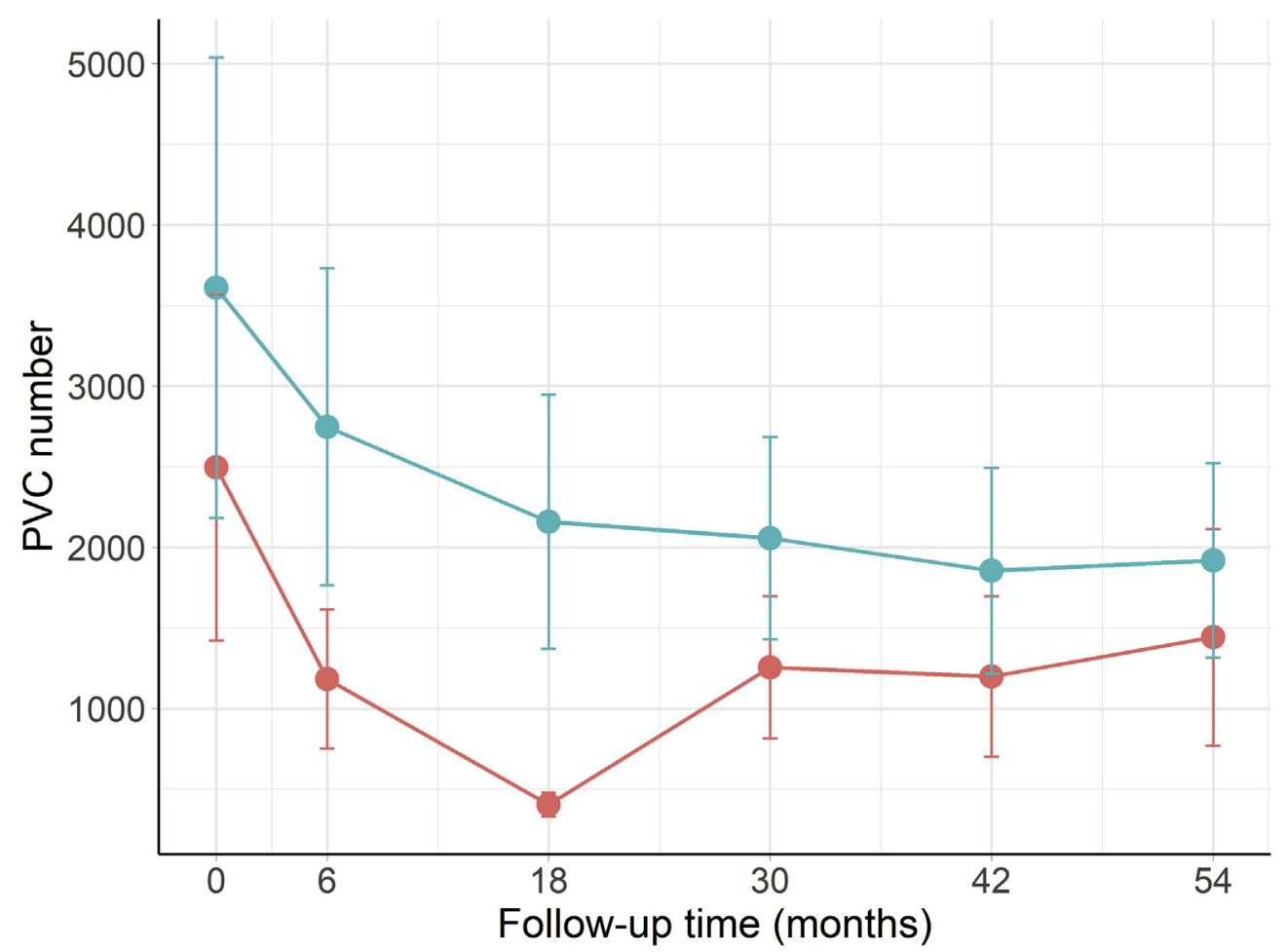

Figure 4 Mean premature ventricular contraction (PVC)/24 hours over follow-up when stratifying for beta-blockers. Teal line indicates patients taking betablocker. Red line indicates patients not taking beta-blocker. No significant difference in trend reduction between the 2 groups was observed. Time is considered from disease diagnosis.

This study sought to present follow-up data from an ARVC cohort of highly characterized athletes undergoing clinical detraining after disease diagnosis and to assess algorithm transferability to an external cohort of high-intensity athletes with ARVC. The enrolled cohort of athletes practiced physical activity almost up until disease diagnosis. All practiced sports consisted of phases of high-end aerobic activity, if not completely endurance sports. Patient population characteristics and clinical results were comparable to those of previously reported cohorts, ${ }^{15,26,27}$ except for mean age, which was due to the small number of master athletes $(n=3)$ that positively skewed our data. The patients in this cohort did not report major variations in training regimen, and the overall training times over the years before

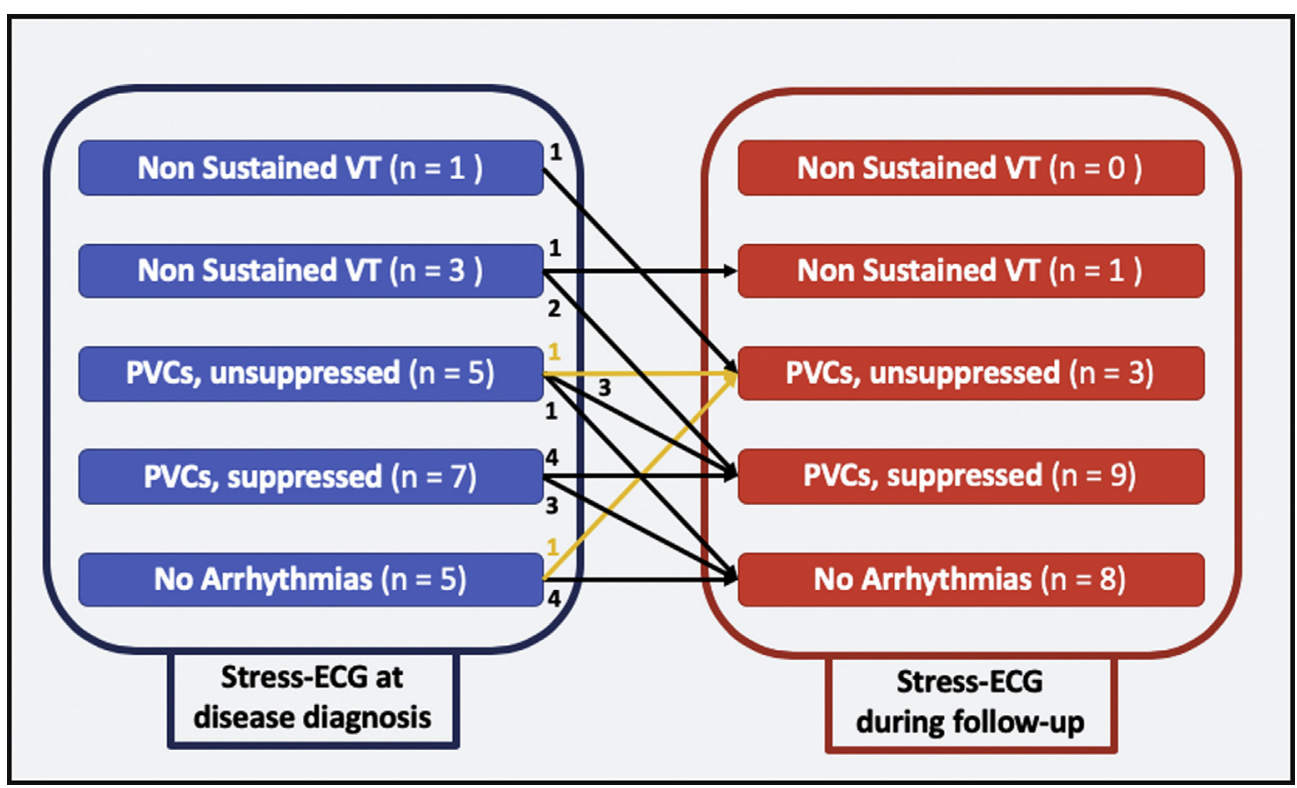

Figure 5 Changes in stress electrocardiogram (ECG) arrhythmic response over time. PVC $=$ premature ventricular complex; VT $=$ ventricular tachycardia. 


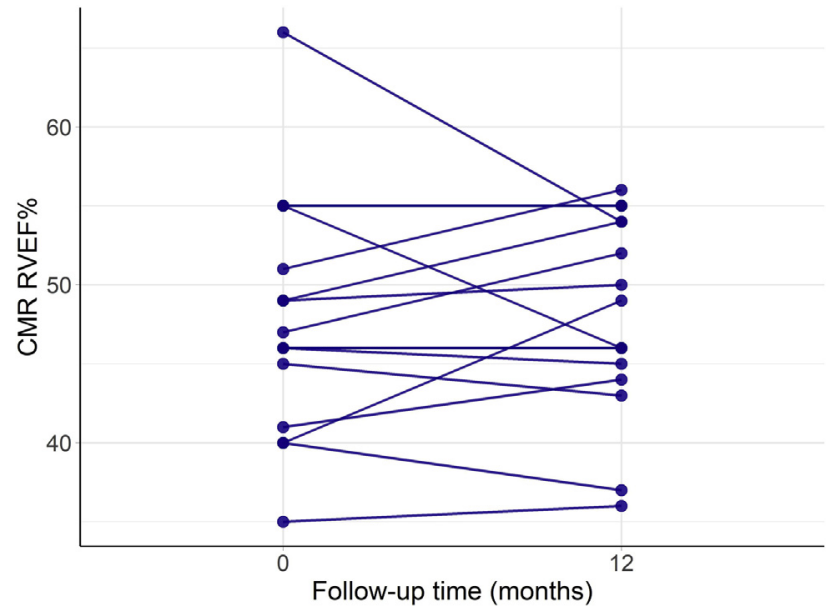

Figure 6 Changes in cardiac magnetic resonance right ventricular ejection fraction (CMR RVEF) over time.

referral and disease diagnosis allowed detailed characterization of the sports history of these patients.

\section{Primary arrhythmic risk evaluation in athletes}

In this cohort, the Cadrin-Tourigny et al algorithm proved reliable in predicting arrhythmic events at all analyzed follow-up points. All patients used for the transferability analysis complied completely with the applicability criteria of the algorithm and were diagnosed with classic ARVC. No gross discrepancies between the a priori and the observed arrhythmic risk were identified in our evaluation. The currently codified clinical parameters seem to account for the increased arrhythmic risk to which athletes are exposed. The impact of exercise on athletes may already be evidenced as lower RVEF and higher PVC counts over 24 hours, leading to higher algorithm-predicted arrhythmic risk.

Of note, our cohort underwent mandatory detraining. Therefore, a potential objection to these results could be that if sport-induced RV remodeling in ARVC patients were fully reversible, at long-term follow-up (eg, at 5 years) this cohort might be more similar to a nonathlete ARVC cohort than to an active athlete (but not yet diagnosed) ARVC cohort from an arrhythmic point of view.

Currently, no proof of complete reversibility of sportinduced remodeling in ARVC patients has been presented. High-end endurance training is associated with worse long-term clinical outcomes, but the mechanisms behind this association are not completely understood nor has the long-term impact of clinical detraining been assessed. ${ }^{15}$ Long-term assessments of ARVC patients who have been practicing endurance sports and who underwent mandatory detraining are limited by both sample size and length of follow-up, so we believe this assessment in a specific ARVC subpopulation to be important for primary risk stratification and for better understanding of the disease. However, even if sport-induced remodeling were to be completely reversible, some time still would be required after implementation of the detraining protocol for it to take effect. In our cohort, the perfect pattern matching between the a priori algorithm-predicted and observed arrhythmic risk was assessed across the entire duration of follow-up, even during the early years when a theoretically regressing but not yet regressed remodeling should be present as an additional factor unaccounted by the algorithm. Larger case series and multicenter studies are needed to further assess the impact and role of both endurance training and clinical detraining in ARVC patients, as well as to completely validate the algorithm in a sportsmen setting. However, data from this first report seem to favor implementation of the algorithm even in this subset of patients.

\section{Impact of clinical detraining on patient characteristics}

Clinical detraining in ARVC athlete patients was associated with a clear reduction in the 24 hour/PVC burden over time. A quantifiable PVC reduction trend is shown in Figure 3. Patients with a higher PVC count generally were also started on beta-blocker therapy, but as shown in Figure 4, both detraining and detraining + beta-blocker management strategy led to similar percentage reductions in PVC burden. Of note, the decrease in PVC burden seems to be maximal within the first 6 months after the detraining interval, plateaus at around 18 months of complete detraining, and remains stable for the duration of follow-up. However, no correlation between the reduction in PVC burden and long-term outcomes has been performed due to the number of patients and trial structure. We agree with Wang et $\mathrm{al}^{15}$ in affirming that clinical detraining is not a strategy aimed at altering ICD placement decision-making but should represent a therapeutic add-on, as also suggested by current international guidelines.

Upon clinical detraining, more than half of the patients who repeated stress ECG reported improvement in arrhythmic findings during follow-up (Figure 5), potentially indicating a role of exercise in progressively self-elicited, exercise-associated arrhythmias.

Although clinical detraining clearly reduced PVC burden and improved arrhythmic stress response, it did not improve RVEF. At 1-year follow-up, in most patients RVEF remained stable, with both clinically and statistically nonsignificant changes. Cessation of physical activity seemed to lead to stabilization of disease progression but not to disease reversal. This result does not seem to contrast the data in a recent study from Chivulescu et al, ${ }^{28}$ who reported progressive deterioration of both morphologic and functional characteristics at CMR of ARVC patients over long-term (median 7.0 years) follow-up. Cessation of physical exercise, the main disease progression factor in our cohort, probably slowed, but did not stop, disease progression. Additional worsening probably would have been detected if morphologic assessment had been performed later (eg, at 5 years). To better assess the impact of clinical detraining on RV contractility, further assessments with large sample sizes and longer follow-up periods are required. Of note, studying the effect of detraining 
on ventricular reverse remodeling in ARVC subpopulations with a genotype more prone to heart failure (eg, desmoglein-2 [DSG-2]) would be of great interest for advancing the entire field.

\section{Outliers not following clinical detraining}

Two patients were classified as outliers because of their refusal to comply with clinical detraining. The first patient met the clinical criteria for ICD implantation in primary prevention but refused to undergo the procedure. The patient kept training regularly and presented at the subsequent follow-up visit with increased PVC burden, several runs of nonsustained ventricular tachycardia on Holter ECG analysis, decreased RVEF, and report of several syncopal events during physical activity. ICD implantation was again proposed, and the patient agreed. Clinical detraining was recommended, and this time the patient complied. Twelve months later, PVC burden was reduced, and RVEF had remained stable.

The second patient, who had been implanted with an S-ICD for primary prevention, refused to comply with clinical detraining for 20 months. The patient continued practicing high-end endurance sports (half-marathon and cycling). After significant deterioration of all clinical parameters had been assessed and after a multidisciplinary consultation that included family members, the patient understood the importance of clinical detraining and agreed to comply. The patient's clinical status remained stable thereafter.

\section{Study limitations}

This study represents the first analysis of a novel ARVC calculator for risk of primary VAs in a cohort of ARVC patients with an extensive athletic background. Study limitations are mostly due to the retrospective nature of the study and the patient follow-up protocol that, although fairly standardized, was not prespecified, possibly introducing some variability to the presented results. The single-center nature of the study may contribute to referral bias, as the entire cohort was extracted from a third-level referral center. In addition, no stratification between gene-positive and geneelusive patients was performed due to the small sample size. Further studies with prospective evaluation and larger sample sizes are needed to completely confirm these results.

\section{Conclusion}

This first analysis of an external cohort seems to validate the use of the Cadrin-Tourigny et al algorithm in athletes. The algorithm seems to account for the practice of high-end endurance sports and does not require specific adjustments. Mandatory clinical detraining has a positive effect on the 24 hour/PVC burden and occurrence of dysrhythmia on stress ECG at mid-term follow-up, with no significant reverse remodeling of RVEF observed. Additional multicenter studies with larger sample sizes are required to confirm the results obtained.

\section{Appendix}

\section{Supplementary data}

Supplementary data associated with this article can be found in the online version at https://doi.org/10.1016/j.hrthm.2020. 03.007 .

\section{References}

1. Corrado D, Link MS, Calkins H. Arrhythmogenic right ventricular cardiomyopathy. N Engl J Med 2017;376:61-72.

2. Akdis D, Brunckhorst C, Duru F, Saguner AM. Arrhythmogenic cardiomyopathy: electrical and structural phenotypes. Arrhythm Electrophysiol Rev 2016; 5:90.

3. Basso C, Bauce B, Corrado D, Thiene G. Pathophysiology of arrhythmogenic cardiomyopathy. Nat Rev Cardiol 2012;9:223-233.

4. Corrado D, Wichter T, Link MS, et al. Treatment of arrhythmogenic right ventricular cardiomyopathy/dysplasia: an international task force consensus statement. Eur Heart J 2015;36:3227-3237.

5. Schuler PK, Haegeli LM, Saguner AM, et al. Predictors of appropriate ICD therapy in patients with arrhythmogenic right ventricular cardiomyopathy: long term experience of a tertiary care center. PLoS One 2012;7. e39584.

6. Al-Ghamdi B, Mallawi Y, Shafquat A, et al. Appropriate and inappropriate implantable cardioverter defibrillators therapies in arrhythmogenic right ventricular cardiomyopathy/dysplasia patients. Cardiol Res 2018;9:204-214.

7. Zorzi A, Rigato I, Bauce B, et al. Arrhythmogenic right ventricular cardiomyopathy: risk stratification and indications for defibrillator therapy. Curr Cardiol Rep 2016;18:57.

8. Platonov PG, Haugaa KH, Bundgaard H, et al. Primary prevention of sudden cardiac death with implantable cardioverter-defibrillator therapy in patients with arrhythmogenic right ventricular cardiomyopathy. Am J Cardiol 2019; 123:1156-1162.

9. Cadrin-Tourigny J, Bosman LP, Nozza A, et al. A new prediction model for ventricular arrhythmias in arrhythmogenic right ventricular cardiomyopathy. Eur Heart J 2019;40:1850-1858.

10. Corrado D, Basso C, Rizzoli G, Schiavon M, Thiene G. Does sports activity enhance the risk of sudden death in adolescents and young adults? J Am Coll Cardiol 2003;42:1959-1963.

11. James CA, Bhonsale A, Tichnell C, et al. Exercise increases age-related penetrance and arrhythmic risk in arrhythmogenic right ventricular dysplasia/cardiomyopathy-associated desmosomal mutation carriers. J Am Coll Cardiol 2013; 62:1290-1297.

12. Kirchhof P, Fabritz L, Zwiener M, et al. Age- and training-dependent development of arrhythmogenic right ventricular cardiomyopathy in heterozygous plakoglobin-deficient mice. Circulation 2006;114:1799-1806.

13. La Gerche A. Defining the interaction between exercise and arrhythmogenic right ventricular cardiomyopathy: editorial. Eur J Heart Fail 2015;17:128-131.

14. Saberniak J, Hasselberg NE, Borgquist R, et al. Vigorous physical activity impairs myocardial function in patients with arrhythmogenic right ventricular cardiomyopathy and in mutation positive family members. Eur J Heart Fail 2014; 16:1337-1344.

15. Wang W, Orgeron G, Tichnell C, et al. Impact of exercise restriction on arrhythmic risk among patients with arrhythmogenic right ventricular cardiomyopathy. J Am Heart Assoc 2018;7. e008843.

16. Marcus FI, McKenna WJ, Sherrill D, et al. Diagnosis of arrhythmogenic right ventricular cardiomyopathy/dysplasia: proposed modification of the task force criteria. Eur Heart J 2010;31:806-814.

17. La Gerche A, Robberecht C, Kuiperi C, et al. Lower than expected desmosomal gene mutation prevalence in endurance athletes with complex ventricular arrhythmias of right ventricular origin. Heart 2010;96:1268-1274.

18. Mitchell JH, Haskell W, Snell P, Van Camp SP. Task Force 8: classification of sports. J Am Coll Cardiol 2005;45:1364-1367.

19. Pelliccia A, Solberg EE, Papadakis M, et al. Recommendations for participation in competitive and leisure time sport in athletes with cardiomyopathies, myocarditis, and pericarditis: position statement of the Sport Cardiology Section of the European Association of Preventive Cardiology (EAPC). Eur Heart J 2019; 40:19-33.

20. Zipes DP, Link MS, Ackerman MJ, Kovacs RJ, Myerburg RJ, Estes NAM. Eligibility and disqualification recommendations for competitive athletes with cardiovascular abnormalities: Task Force 9: arrhythmias and conduction defects: a scientific statement from the American Heart Association and American College of Cardiology. Circulation 2015;132:e315-e325.

21. Sharma S, Merghani A, Mont L. Exercise and the heart: the good, the bad, and the ugly. Eur Heart J 2015;36:1445-1453. 
22. Ruwald A-C, Marcus F, Estes NAM, et al. Association of competitive and recreational sport participation with cardiac events in patients with arrhythmogenic right ventricular cardiomyopathy: results from the North American multidisciplinary study of arrhythmogenic right ventricular cardiomyopathy. Eur Heart J 2015;36:1735-1743.

23. Coelho S, Silva F, Silva J, António N. Athletic training and arrhythmogenic right ventricular cardiomyopathy. Int J Sports Med 2019;40:295-304.

24. Lie $\varnothing \mathrm{H}$, Dejgaard LA, Saberniak J, et al. Harmful effects of exercise intensity and exercise duration in patients with arrhythmogenic cardiomyopathy. JACC Clin Electrophysiol 2018;4:744-753.

25. Casella M, Gasperetti A, Gaetano F, et al. Long-term follow-up analysis of a highly characterized arrhythmogenic cardiomyopathy cohort with classical and non-classical phenotypes-a real-world assessment of a novel prediction model: does the subtype really matter. Europace 2020. euz352.

26. Calore C, Zorzi A, Sheikh N, et al. Electrocardiographic anterior T-wave inversion in athletes of different ethnicities: differential diagnosis between athlete's heart and cardiomyopathy. Eur Heart J 2016;37:2515-2527.

27. Brosnan MJ, te Riele ASJM, Bosman LP, et al. Electrocardiographic features differentiating arrhythmogenic right ventricular cardiomyopathy from an athlete's heart. JACC Clin Electrophysiol 2018;4:1613-1625.

28. Chivulescu M, Lie $\varnothing \mathrm{H}$, Popescu BA, et al. High penetrance and similar disease progression in probands and in family members with arrhythmogenic cardiomyopathy. Eur Heart J 2019. ehz570. 